\title{
Global microscopic nuclear level densities within the HFB plus combinatorial method for practical applications
}

\author{
S. Hilaire ${ }^{1, a}$, S. Goriely ${ }^{2}$, and A.J. Koning ${ }^{3}$ \\ 1 CEA/DAM Île-de-France, DPTA/Service de Physique Nucléaire, BP. 12, 91680 Bruyères-le-Châtel, France \\ 2 Institut d'Astronomie et d'Astrophysique, Université Libre de Bruxelles, Belgium \\ ${ }^{3}$ Nuclear Research and Consultancy Group NRG, PO. Box 25, 1755 ZG Petten, The Netherlands
}

\begin{abstract}
New energy-, spin- and parity-dependent nuclear level densities based on the microscopic combinatorial model are proposed for practical applications. The combinatorial model includes a detailed microscopic calculation of the intrinsic state density and of the rotational enhancement factor, but a phenomenological treatment of the vibrational effect. The calculations make a coherent use of nuclear structure properties determined within the deformed SkyrmeHartree-Fock-Bogolyubov framework. The present model predicts the experimental s-wave and p-wave neutron resonance spacings with a degree of accuracy comparable to that of the best global models available. It is also shown that the model gives reliable extrapolations to low energies where experimental data on the cumulative number of levels can be extracted. Level densities for more than 8500 nuclei are made available in a table format for practical applications.
\end{abstract}

\section{Introduction}

The knowledge of nuclear level densities (NLDs) has been a matter of interest and study for more than 50 years, going back at least to 1936 with Bethe's pionnering work [1]. Since then, more or less sophisticated methods have been developped to reproduce the available experimental data $[2,3]$. For applications such as astrophysics or accelerator driven systems, which require a large number of data far away from the experimentally known regions, extrapolations as reliable and accurate as possible have to be done. If analytical expressions for NLDs are used, the parameters on which they depend have to be extrapolated without having any way to check the quality of these extrapolations. Therefore, one should preferentially use microscopic or semi-microscopic global predictions, provided they compete with more phenomenological highlyparameterized formulae for the reproduction of experimental data. The microscopic character indeed ensures the required reliability, while the searched accuracy is garanted by the ability to reproduce experimental data as well as the best global phenomenological approaches do.

Many global microscopic approaches have been developed in the past but they are almost never used for practical applications, either because of their lack of accuracy or because they do not offer the same flexibility as the analytical expressions which can be easily adjusted. Recently, we have performed large scale calculation of NLDs for nearly 8500 nuclei, modernizing the combinatorial method described in ref. [3] which had shown its predictive power $[3,4]$. The main advantage of our results is to provide not only the NLDs tabulated as function of the excitation energy, but also the spin and parity distributions without any statistical assumption.

After summarizing the method used to compute these NLDs, we show how our combinatorial results can compete with global analytical expressions when compared with experimental data. We then discuss the parity and spin distributions

${ }^{a}$ Presenting author, e-mail: stephane.hilaire@cea.fr and show their impact on selected examples. We finally explain how the flexibility usually claimed to be the main advantage of analytical approaches is also possible with our tables.

\section{The combinatorial method}

As precisely described in ref. [2], our method consists in using the single-particle level schemes obtained from constrained axially symmetric Hartree-Fock-Bogoliubov (HFB) method to construct incoherent particle-hole state densities $\omega(U, K, \pi)$ as functions of the excitation energy $U$, the spin projection (on the intrinsic symmetry axis of the nucleus) $K$ and the parity $\pi$. Once these incoherent particle-hole state densities are determined, we then account for collective effects, namely the vibrational enhancement, and, for deformed nuclei, the rotational enhancement factors, to deduce total level densities $\rho(U, J, \pi)$ (function of the spin $J$ rather than the spin projection $K$ ) which can then be compared with experimental data.

If the nucleus under consideration displays spherical symmetry, $\rho(U, J, \pi)$ is trivially obtained through the relation

$$
\rho(U, J, \pi)=\omega(U, K=J, \pi)-\omega(U, K=J+1, \pi),
$$

while for deformed nuclei, rotational bands are explicitely constructed to get the deformed level density. In this last case, any intrinsic state $K^{\pi}$ is the band head of a set of levels having the same parity and spins $J=K, K+1, K+2, \ldots$ if $K \neq 0$ or $J=0,2,4, \ldots$ (resp. $J=1,3,5, \ldots$ ) if $K=0^{+}$(resp. $K=0^{-}$). These levels form rotational bands in which the energy of each member can be deduced from the band-head energy provided the difference $E(J, K)$ between the energy of the level $J^{\pi}$ and that of the band-head state $K^{\pi}$ is known. In our approach, this rotational energy is obtained with the well-known expression [5],

$$
E(J, K)=1 / 2\left(J^{2}+J-K^{2}\right) / \mathcal{J} .
$$




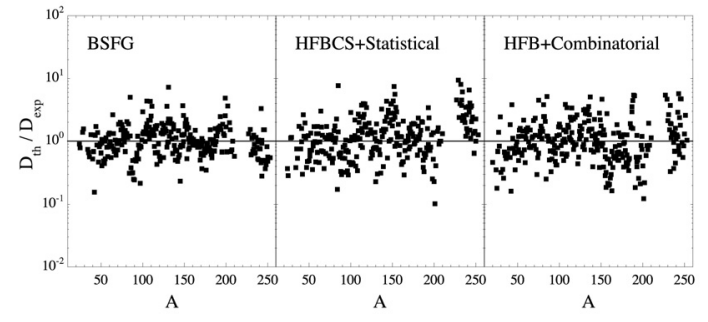

Fig. 1. Ratio of theoretical $D_{t h}$ BSFG [8] (left), HFBCS plus statistical [7] (center) and the present HFB+combinatorial (right) to experimental $D_{\text {exp }}$ s-wave mean neutron resonance spacings for the 295 nuclei compiled in ref. [9].

where $\mathcal{J}$ is the moment of inertia of a nucleus rotating around an axis perpendicular to the symmetry axis which, in our case, takes the rigid body value of an ellipsoidal shape.

Once this transition from state to level densities is done, vibrational effects are taken into account multiplying $\rho(U, J, \pi)$ by the vibrational enhancement $K_{v i b}$ approximated by the phenomenological expression described in refs. [2] and [6] which mainly depends on the quadrupole and octupole vibrational energies. The main originality of our treatment comes from the fact that we have fitted these energies using an empirical expression which contains a shell correction term [2].

Finally, when the excitation energy increases, it is well known that deformed nuclei tend to become spherical. Such a shape transition significantly affects the NLDs predictions. As suggested in ref. [7], this effect is described by introducing a phenomenological damping function between the spherical and deformed expressions of the nuclear level densities.

\section{Comparisons with experimental data}

Despite the considerable experimental efforts made to derive NLD data, the lack of reliable data - especially over a wide energy range - constitutes the main problem that NLD theories have to face. Beyond the slow neutron resonances data and the cumulative numbers of low energy levels, other sources of information have also been suggested, such as analyses of spectra of evaporated particles and coherence widths of cross section fluctuations. However, these experimental data are affected by systematic errors resulting from experimental uncertainties as well as from the use of approximate theories to analyse them. In fact only the experimental data from the s-wave and p-wave neutron resonance spacings and the compilation of low energy observed excited levels represent reliable source of information on NLDs. Note, however, that different compilations of resonance spacings show non-negligible differences, especially for spherical nuclei, for which only a few neutron resonances are observed.

Starting with the s-wave neutron resonance spacings, we show in figure 1 the results of our approach with respect to experimental data (right panel). Globally, the predictions remain within a factor of 2 with no deviations larger than a factor of 8 being found. In the same figure, are also shown the predictions obtained with an accurate global BSFG-type formula [8] as well as those of the HFBCS plus statistical estimate of ref. [7]. Figure 1 clearly shows that the present

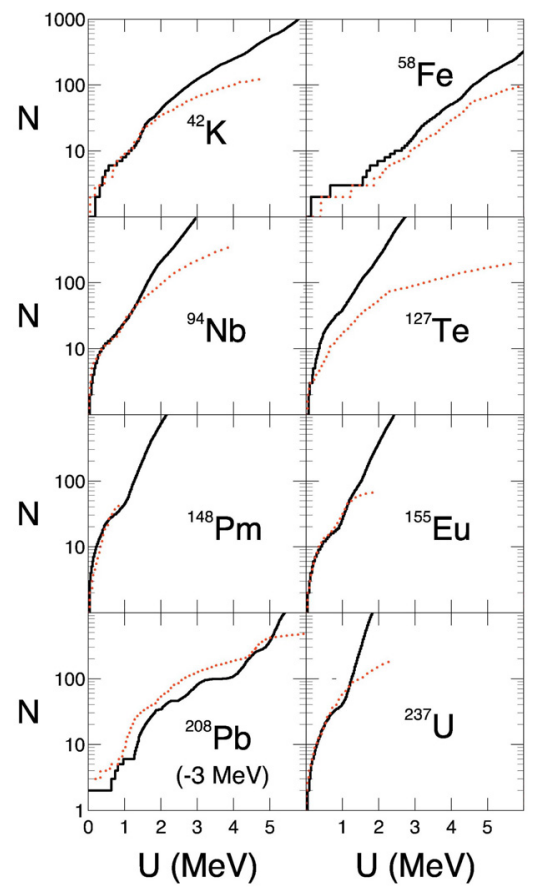

Fig. 2. Comparison of the cumulative number of observed levels (dotted staircase) with the HFB plus combinatorial predictions (solid lines) as a function of the excitation energy $U$ for a sample of 8 nuclei. For ${ }^{208} \mathrm{~Pb}$, both curves have been shifted by $3 \mathrm{MeV}$, the energy range corresponding consequently to [3-9] MeV instead of [0-6] MeV.

approach is comparable to the previous predictions, but also that no systematic deviations is obtained.

When looking at lower energies, we observe that our model also gives quite good results. As an example, in figure 2 we compare the predicted cumulative number of levels $N(U)$ with the experimental data [9] for 8 nuclei, including light as well as heavy, spherical as well as deformed species.

As discussed above, the experimental spacings are predicted within roughly a factor of 2 , so that a similar factor could affect the cumulative number of levels. As will be discussed in section 5 , it is however possible to renormalize the present microscopic NLD on both the experimental level scheme at low energy and on the neutron resonance spacings. Note that in many of these cases, as for instance in the case of ${ }^{94} \mathrm{Pm}$, a well-defined structure can be observed both for the experimental and theoretical plots. Such a structure is due to the complex interplay of shell, pairing and deformation effects at low energies. Only a microscopic model, such as the present one, can possibly describe such complex low-energy patterns. In particular, the so called "constant-temperature" formula, widely used to extrapolate NLDs at low energies, inevitably fails to reproduce such patterns and therefore to estimate reliably the number of levels up to which the experimental level scheme is complete, a quantity of particular relevance in reaction codes $[9,10]$.

\section{Impact of realistic spin/parity distributions}

Apart from the previous quantitative analysis, it is also interesting to pay attention to the spin and parity distributions 

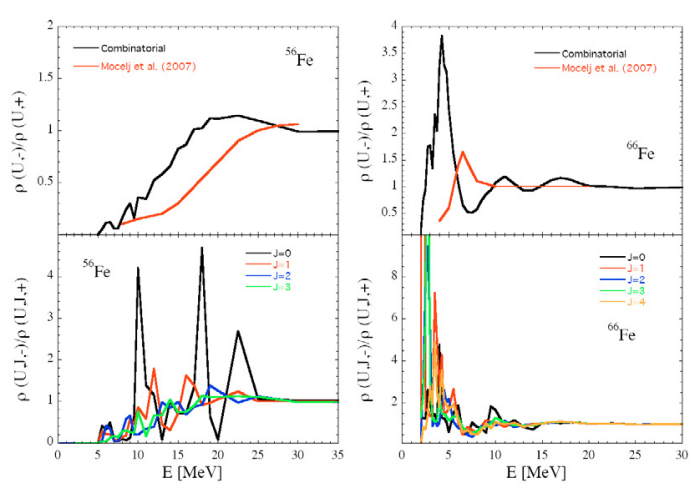

Fig. 3. Ratio of the total parity dependent level densities (top) and for few selected spin values (bottom) as function of the excitation energy.

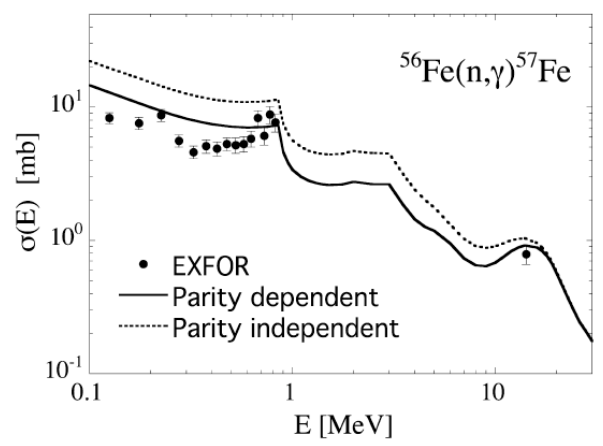

Fig. 4. ${ }^{56} \mathrm{Fe}(\mathrm{n}, \gamma){ }^{57} \mathrm{Fe}$ cross section with the parity-dependent nuclear level densities and the parity-independent one obtained avering both parities.

obtained in our approach. As shown in figure 3, the simultaneous treatment of parities and spins reveals the complexity of the level densities.

Indeed, while in the case of ${ }^{56} \mathrm{Fe}$ the ratio of total NLDs $\rho(U, \pi=-) / \rho(U, \pi=+)$ seems to be quite smooth, the same ratio for specified spin values shows strongly oscillating structures. The situation is even more pronounced for ${ }^{66} \mathrm{Fe}$. In the same figure, we show the results of ref. [11] using the assumption of Poisson distributed independent quasi-particles. It is clear that we find much more complex patterns, since our approach contains microscopic subtleties that cannot be mimicked by a too simple Poisson law. It is also worth noticing that even for excitation energies close to the neutron binding energy, the usually adopted parity equipartition hypothesis is not really valid.

Also interesting is the impact of realistic parity distributions on cross section calculations, illustrated on the ${ }^{56} \mathrm{Fe}$ radiaitive neutron capture in figure 4 . In this figure, the cross section with the parity-dependent NLD is compared with the cross section obtained assuming the NLD to be given by the average value of both parities. This comparison clearly illustrates that in cross section calculations, especially for light nuclei, the full parity distribution of the NLD can have a direct impact on the predictions.

The spin distribution obtained within the combinatorial approach can also deviate from a Gaussian distribution, especially at low energy. This deviation can significantly

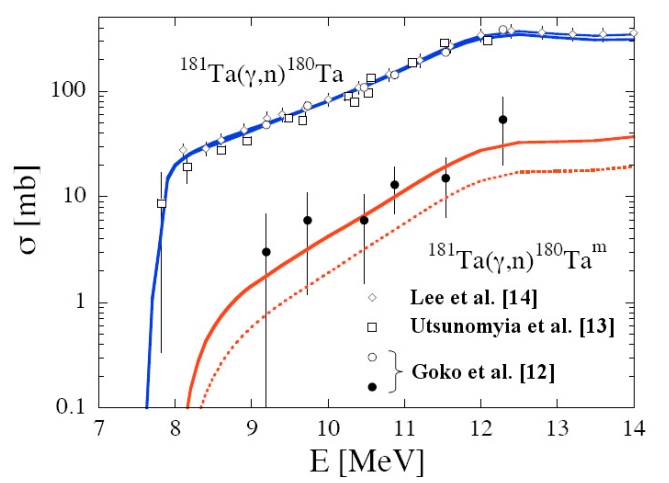

Fig. 5. Comparison between experimental and theoretical cross sections. Open symbols correspond to the total photoreaction [12-14] and solid symbols to the partial cross section for the ${ }^{181} \mathrm{Ta}(\gamma, \mathrm{n})^{181} \mathrm{Ta}^{\mathrm{m}}$ only [12]. The solid line is obtained with our tables while the dashed line is obtained using the level densities of ref. [7].

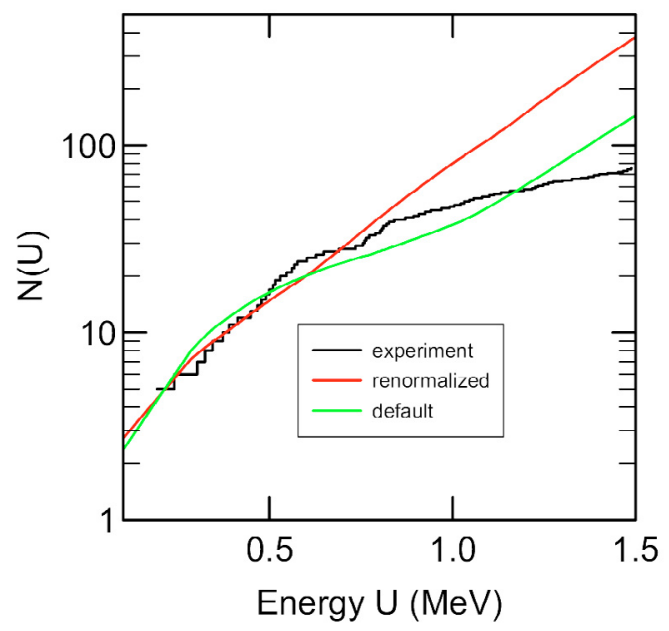

Fig. 6. Comparison of the experimental cumulative number of observed levels with our results with and without renormalization for ${ }^{239} \mathrm{Pu}$.

affect some reaction channel, like the isomeric to ground states production ratio in the ${ }^{181}$ Ta photoneutron reaction [12], illustrated in figure 5.

In this case, it was found that the combinatorial model predicts more high-spin states at low energy than a Gaussian distribution would, leading to a significantly larger production of the $9^{-}$isomeric state of ${ }^{180} \mathrm{Ta}$, in better agreement with experimental data.

\section{Renormalization procedure}

In this section, we would like to mention that for many nuclear physics applications, a renormalization procedure of the NLD on experimental data (such as the s-neutron spacings or the low-lying levels) is required, in particular for nuclear data evaluation or for an accurate and reliable estimate of reaction cross sections. Even if our combinatorial NLD are provided in a table format, it is possible to 
renormalize them on both the experimental level scheme at low energy and the neutron resonance spacings in a way similar to what is usually done with analytical formulae. More precisely, the tabulated level densities can be corrected writing

$$
\rho_{\text {renorm }}(U, J, \pi)=e^{\sqrt{\alpha(U-\delta)}} \times \rho(U-\delta, J, \pi)
$$

where the energy shift $\delta$ can be adjusted to fit the cumulative number of levels and $\alpha$ is tuned to reproduce the experimental s-wave neutron spacing. Note however, that the tables provided have not been renormalized.

To illustrate such adjustement, we show in figure 6, the default and adjusted cumulated histograms. As expected, the renormalization procedure allows for a better description of the low energy discrete levels.

\section{Conclusions}

Tabulated level densities have been determined for nearly 8500 nuclei and can be downloaded from the web at the address http://www-astro.ulb.ac.be/Html/nld_comb.htm. They have the same level of quality of usually employed global analytical expressions with the advantage of being (i) more reliable because of their microscopic grounds and (ii) spin and parity dependent. Also a recipe is given to adjust the tables as one plays with the parameters of the analytical expressions when fitting experimental cross section for evaluation purposes.

\section{References}

1. H.A. Bethe, Phys. Rev. 50, 332 (1936).

2. S. Hilaire, S. Goriely, Nucl. Phys. A 779, 63 (2006).

3. S. Hilaire, J.P. Delaroche, M. Girod, Eur. Phys. J. A 12, 169 (2001) and references therein.

4. S. Goriely, in Proceedings of the International Conference on Frontiers in Nuclear Structure, Astrophysics and Reactions, 2006 edited by S. Harissopulos, P. Demetriou, R. Julin, AIP Conference Proceedings 831 (New York, 2006), p. 8.

5. T. Dossing, A.S. Jensen, Nucl. Phys. A 222, 493 (1974).

6. A.V. Ignatyuk, IAEA report, TECDOC-1034, 1998.

7. P. Demetriou, S. Goriely, Nucl. Phys. A 695, 95 (2001).

8. S. Goriely, J. Nucl. Sci. Technol. Suppl. 2, 536 (2002).

9. T. Belgya et al., in Handbook for calculations of nuclear reaction data, Reference Input Parameter Library-2, IAEA-TECDOC1506 (IAEA, Vienna, 2006).

10. A.J. Koning, S. Hilaire, M.C. Duijvestijn (this proceedings).

11. D. Mocelj et al., Phys. Rev. C (in press).

12. S. Goko et al., Phys. Rev. Lett. 96, 192501 (2006).

13. H. Utsunomiya et al., Phys. Rev. C 67, 015807 (2003).

14. Lee et al., IAEA-TECDOC-1178 (2000). 